\title{
AN ANOMALOUS EXTENSOR INDICIS MUSCLE
}

\author{
B. Victor Jones. R.N., Ipswich, SUffolk, England
}

In the case described below the extensor indicis tendon was replaced by a short muscle arising from the dorsum of the wrist. simulating a ganglion.

A naval cook, aged twenty, complained of diffuse pain in the left hand after heavy use. with a swelling on the back of the hand. He had first noticed this swelling three years before. and said that it had appeared spontaneously. A course of short-wave diathermy had failed to relieve his symptoms.

Examination showed an elongated tumour on the dorsum of the hand, which appeared to arise from the extensor tendons to the index finger and passed upwards under the extensor retinaculum of the wrist (Fig. 1). It was soft. semi-fluctuant and slightly tender. It was noted that it was not translucent, but the significance of this observation was not realised at the time.

The appearance of the right hand was normal. but on palpation a slight thickening was felt in the line of the extensor tendons to the index finger. On questioning he admitted to occasional pain on the back of this hand also.

The tumour was diagnosed as a ganglion and was explored. At operation it was found to be a muscle replacing the extensor indicis tendon. This muscle arose from the soft tissues on the dorsum of the wrist under cover of the extensor retinaculum. At the neck of the second

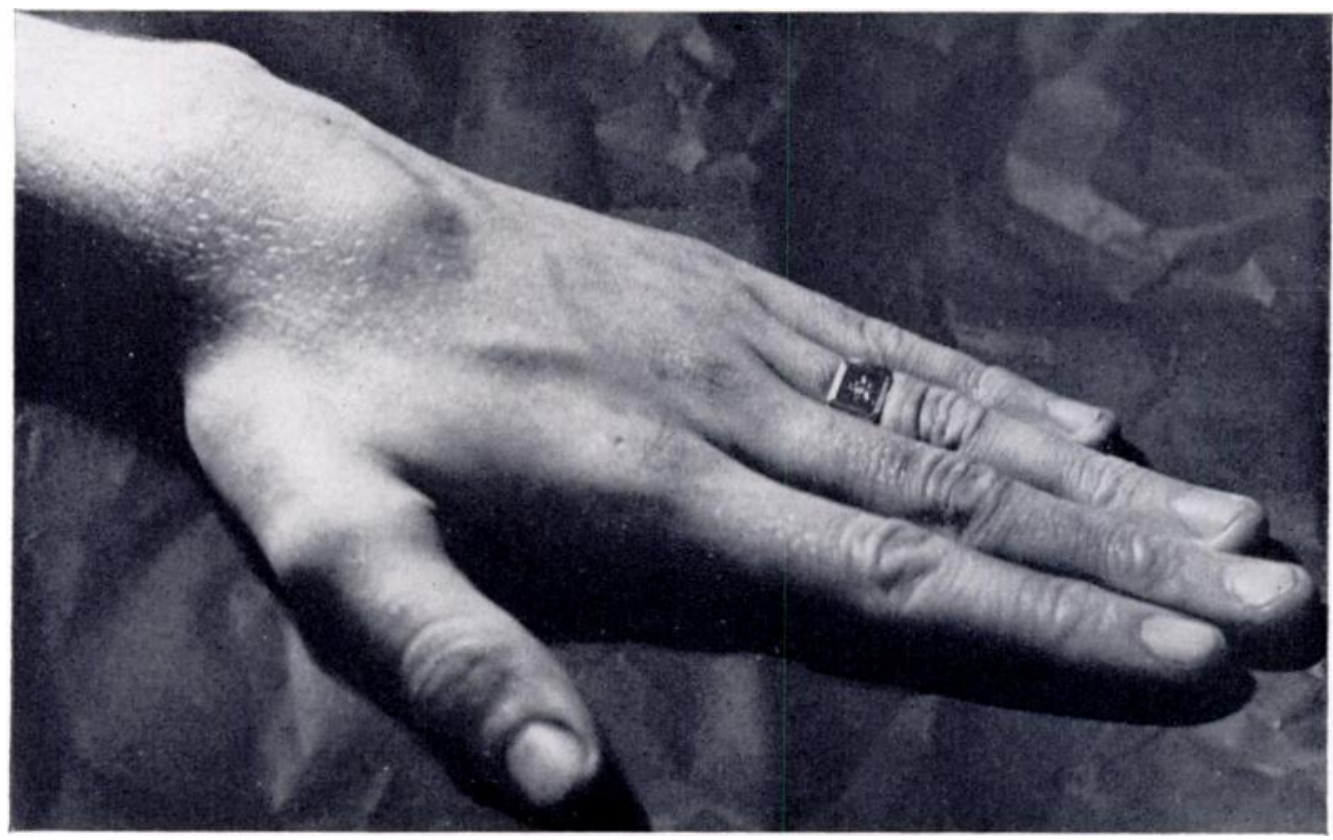

Fig. 1

The hand at rest. The tumour became more prominent when the index finger was extended against resistance.

metacarpal bone it became tendinous and joined the ulnar side of the tendon of extensor digitorum communis to the index finger to form the extensor expansion (Fig. 2). From the origin of the muscle a thin fibrous band ran upwards into the forearm along the line which the extensor indicis tendon would have been expected to take. This band was not mobile and showed no evidence of muscle fibres. 
It was considered probable that the cause of his symptoms was constriction of the muscle by the extensor retinaculum, which was therefore divided. Since the operation the symptoms have improved considerably, but he still has some aching in the muscle after, rather than during, heavy use of the hand.

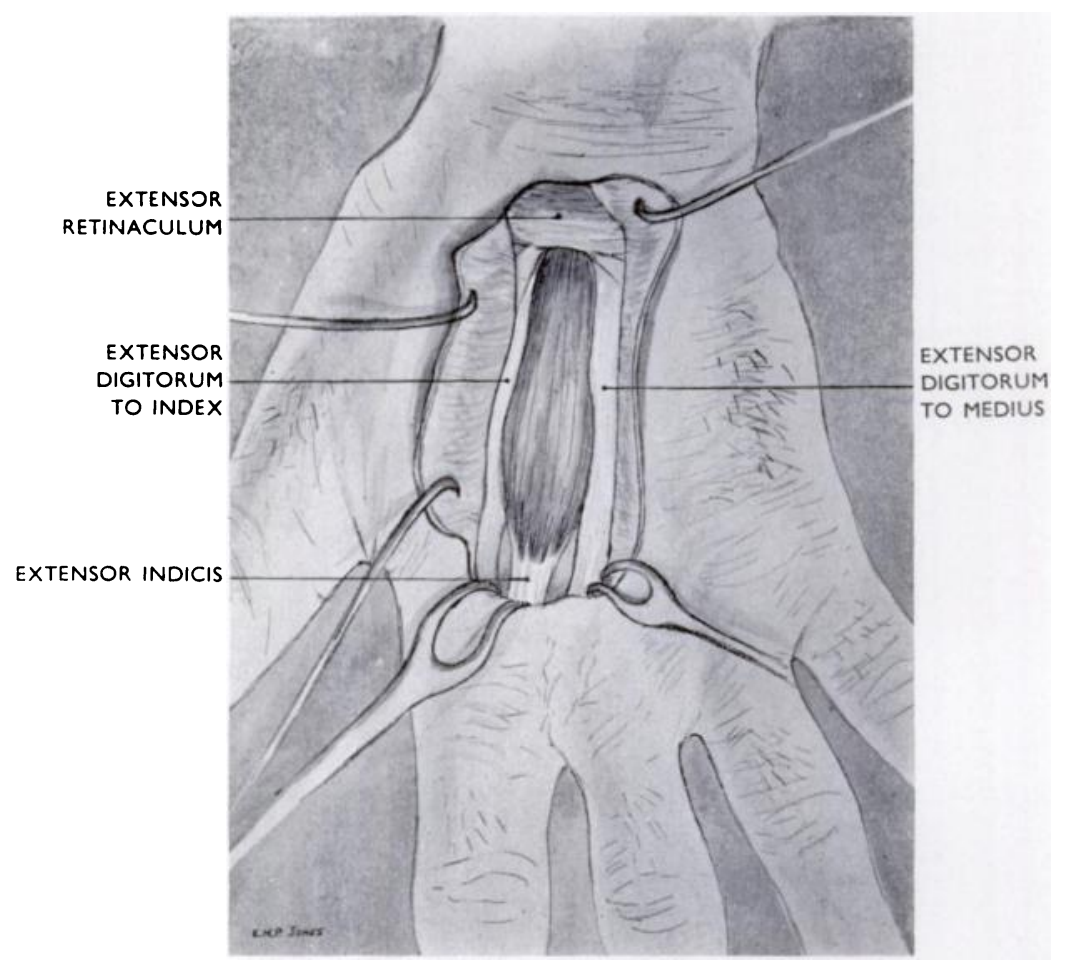

FiG. 2

Drawing made trom a photograph taken at operation, showing relationship of the muscle to the tendons of extensor digitorum communis.

\section{DISCUSSION}

In amphibia the digits are controlled solely by intrinsic muscles, an extensor digitorum brevis muscle being situated on the dorsum of the manus. In man this muscle has disappeared in the upper limbs. its functions being taken over by forearm muscles with long tendons to the digits. Occasionally, atavistic muscles representing parts of the old extensor brevis are found.

The extensor indicis muscle normally arises from the posterior surface of the shaft of the ulna, below the origin of the extensor pollicis longus, and from the interosseous membrane. Its tendon passes under the extensor retinaculum in company with the tendons of extensor digitorum communis, joining the ulnar side of the communis tendon to the index finger opposite the head of the second metacarpal bone.

Cauldwell, Anson and Wright (1943), in a study of the extensor indicis muscle in 263 consecutive specimens, found three cases in which the muscle had an abnormal origin. In one case the muscle had the usual origin from the ulna, became tendinous, and then again became muscular with a secondary attachment in the region of the carpal bones. In the second case a muscle with a rudimentary origin at the normal site was inserted into a second muscle arising from two heads from the proximal carpal bones. In the third case a short muscle only was present, arising from the distal end of the radius, proximal carpal bones and related ligaments. They called this short muscle "extensor digiti brevis manus," and stated that it may have from 
one to four tendons, though a single tendon to the index or middle finger is the most common arrangement. Typically this muscle arises from the distal end of the radius and ulna, from the carpal bones and from the associated ligaments; rarely it arises from the metacarpus or the dorsal carpal ligament.

Bunnell (1956) encountered three patients who had either one or two slips of atavistic extensor digitorum brevis muscle in the hand. He considered that phylogenetically the forearm extensors of the amphibia became secondarily attached to the bellies of the extensor brevis, which then became tendinous and lost their attachment to the carpus.

Anomalous muscles may be confused with ganglia or with other soft-tissue tumours: the palmaris longus with muscle fibres extending down into the palm and simulating a compound palmar ganglion is a familiar example. They are among those conditions of which it may be said: " to think of it is to make the diagnosis." In this case the diagnosis could have been made by noting the lack of translucency of the tumour and its hardening and increase in size when the finger was extended against resistance.

This case illustrates that when such muscles are constricted by normal retinacula under which they pass they may cause symptoms. Division of the retinaculum gave considerable relief in this case.

\section{SUMMARY}

1. A case is described in which the extensor indicis tendon was replaced by a short muscle arising from the soft tissues on the dorsum of the wrist.

2. The patient complained of pain in the hand after heavy use. This was considered to be due to constriction of the muscle by the extensor retinaculum. Division of the retinaculum was followed by a considerable improvement in his symptoms.

I wish to thank the Medical Director-General of the Navy for permission to publish this report.

\section{REFERENCES}

Bunnell, S. (1956): Surgery of the Hand. Third edition, p. 4. London: Pitman Medical Publishing Co. Ltd. Cauldwell, E. W., Anson, B. J., and Wright, R. R. (1943): The Extensor Indicis Proprius Muscle. A Study of 263 Consecutive Specimens. Quarterly Bulletin of Northwestern University Medical School, 17, 267. 